\title{
Low-dose post-transplant cyclophosphamide and anti-thymocyte globulin as an effective strategy for GVHD prevention in haploidentical patients
}

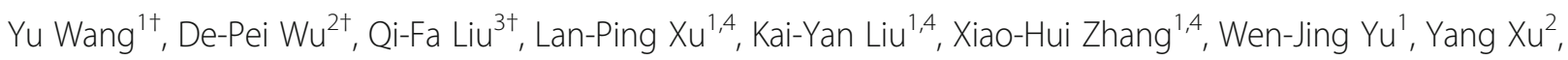
Fen Huang ${ }^{3}$ and Xiao-Jun Huang ${ }^{1, *^{*}}$ (D)

\begin{abstract}
Background: Low-dose post-transplant cyclophosphamide (PTCy) in conjunction with anti-thymocyte globulin (ATG) appears as a potentially effective graft-versus-host disease (GVHD) prevention strategy in haploidentical hematopoietic cell transplant (haplo-HCT). Our study aims to assess the efficacy of this regimen.

Methods: We extended our prospective study in patients treated with low-dose PTCy (14.5 mg/kg on days 3 and 4 ) in ATG/granulocyte colony-stimulating factor (G-CSF)-based regimen and compared the results to the contemporary cohort of patients without low-dose PTCy (ATG cohort). Both study cohort and control are transplanted from maternal donor or collateral relatives.

Results: We identified 239 consecutive patients (ATG-PTCy cohort = 114; ATG cohort = 125). All patients but one in ATG cohort achieved myeloid engraftment by day 30 post-HCT. We found that both the cumulative incidence of 100-day grade III-IV aGVHD and non-relapse-mortality (NRM) in the ATG-PTCy cohort was significantly reduced than that in the ATG group (5\% vs 18\%; $P=0.003$; and 6\% vs 15\%; $P=0.045)$; the 2 -year cumulative incidences of relapse and overall survival were comparable between the two cohorts (13\% vs $14 \% ; P=0.62$; and $83 \%$ vs $77 \% ; P=0.18$, respectively). Furthermore, GVHD-free, relapse-free survival (GRFS) was significantly improved in the ATG-PTCy arm (63\% vs 48\%; $P=$ 0.039). In multivariate analysis, the joint treatment resulted in lower grade II-IV acute GVHD (HR 0.58; $P=0.036$ ), grade IIIIV aGVHD (HR 0.28; $P=0.006$ ), chronic GVHD (HR 0.60; $P=0.047)$, NRM (HR 0.26; $P=0.014)$, and higher GRFS (HR 0.59; $P=$ 0.021 ) but slower myeloid and platelet recovery (HR 0.29 and 0.30; both $P<0.001$ ).
\end{abstract}

Conclusions: These results suggested that ATG/PTCy (low-dose) can reduce both acute and chronic GVHD as compared with standard ATG-based prophylaxis using maternal donor or collateral relatives at particular high GVHD risk.

Keywords: Anti-thymocyte globulin, Post-transplant cyclophosphamide, Low-dose, Haploidentical, Graft-versus-host disease

\footnotetext{
* Correspondence: xjhrm@medmail.com.cn

${ }^{\dagger}$ Yu Wang, De-Pei Wu and Qi-Fa Liu contributed equally to this work.

${ }^{1}$ Peking University People's Hospital, Peking University Institute of

Hematology, National Clinical Research Center for Hematologic Disease,

Beijing Key Laboratory of Hematopoietic Stem Cell Transplantation, No.11,

Xizhimen South Street, Xicheng District, Beijing 100044, China

${ }^{4}$ Peking-Tsinghua Center for Life Sciences, Beijing 100871, China

Full list of author information is available at the end of the article
}

(c) The Author(s). 2019 Open Access This article is distributed under the terms of the Creative Commons Attribution 4.0 International License (http://creativecommons.org/licenses/by/4.0/), which permits unrestricted use, distribution, and reproduction in any medium, provided you give appropriate credit to the original author(s) and the source, provide a link to the Creative Commons license, and indicate if changes were made. The Creative Commons Public Domain Dedication waiver (http://creativecommons.org/publicdomain/zero/1.0/) applies to the data made available in this article, unless otherwise stated. 


\section{Background}

During the past 20 years, wider application of easily available haploidentical donor hematopoietic cell transplant (haplo-HCT) has been made possible through the $\mathrm{T}$ cell-replete (TCR) regimens including $\mathrm{T}$ cell regulation with anti-thymocyte globulin (ATG)/granulocyte colony-stimulating factor (GCSF) and post-transplant cyclophosphamide (PTCy) [1-9]. To achieve decreased non-relapse mortality (NRM) and improved long-term outcomes in haploidentical transplant, the joint use of ATG and PTCy might effectively reduce graft-versushost disease (GVHD) and mortality associated with severe forms of GVHD [10-14]. There are multiple papers using ATG/PTCy (high-dose) in patients with Fanconi anemia, aplastic anemia, and sickle cell disease [10]. To date, the combination of conditioning regimens with lowdose ATG and high-dose PTCy after haplo-HCT [11-14] or unrelated donor (URD) HCT $[15,16]$ for hematological malignancies has been documented in several reports with reduced rates of GVHD and acceptable relapse rate albeit with somewhat high rates of graft failure (8\%) [11] or delayed engraftment [12]. Recently, results of a prospective trial have been available which analyze the efficacy of combined use of low-dose ATG and 1-day high-dose PTCy $(50 \mathrm{mg} / \mathrm{kg}$ at +3 days) in preventing GVHD among haplo patients [17].

Recently, we established a regimen using low-dose PTCy in conjunction with standard-dose ATG in order to lower the risk of GVHD without compromising engraftment and disease relapse. This protocol consisted of a myeloablative conditioning (MAC) regimen containing cytarabine, busulfan, Cy combined with standard-dose ATG/G-CSF (the socalled Beijing protocol), and followed by low-dose PTCy $(14.5 \mathrm{mg} / \mathrm{kg}$ on days 3 and 4 after HCT) for haplo-HCT recipients. We previously reported this strategy in preliminary prospective study wherein reduced incidences of both acute and chronic GVHD were observed in comparison with non-combined regimens [18]. In this prospective trial, results indicated that low-dose PTCy is sufficient to lower acute GVHD in mouse model, partly due to the boosting of fast regulatory $\mathrm{T}$ cell (Treg) reconstitution. In addition, low-dose PTCy could augment the protective effect of ATG on GVHD both in mouse and human sets while ensuring high rates of engraftment and keeping disease control [18]. These results seem to highlight the feasibility of this novel procedure with low-dose PTCy in ATG-based MAC for GVHD prevention and perfect outcomes post-haplo-HCT. However, the potentially effective regimen needs to be validated on a larger population with hematological neoplasms.

With the aim to assess the efficacy of this regimen in haplo-HCT, we extended our prospective study in consecutive patients treated with ATG/GCSF-PTCy (lowdose) regimen and initiated a multicenter analysis on comparing the results to the contemporary cohort of patients who received ATG/GCSF without low-dose PTCy. In the current study, patients in both study group and contemporary control group are all transplanted from maternal donor (mother donors, MDs) [3] or collateral relative donors (CRDs, e.g., uncle, aunt, nephew, niece, and cousins) $[19,20]$ who had especially high risk of GVHD occurrence under "Beijing protocol." Herein, we tested the efficacy of ATG/PTCy (low-dose) as compared with standard ATG-based prophylaxis using maternal donor or collateral relatives. Thus, this new approach in unmanipulated haplo-HCT with low-dose PTCy in ATG/ GCSF-based MAC would expand the donor pool for patients eligible for allogeneic HCT, by allowing the safe and effective use of specific haploidentical donors associated with especially high risk of GVHD occurrence.

\section{Patients and methods}

\section{Inclusion and exclusion criteria}

Eligibility criteria included consecutive patients with hematological neoplasms, who underwent a first alloHCT from April 2015 (start time of the clinical trial) and extended to June 2018, using either MDs or CRDs. Signed informed consent was given to all patients and donors. The study protocol was approved by the Institutional Review Board of each center and was in accordance with the Declaration of Helsinki. Patients were defined as having low-, intermediate-, or high-risk disease stages according to the established disease risk index [21, 22]. Exclusion criteria were contraindications to HCT such as severe heart, kidney, or liver function failure and a prior transplant.

\section{Donors}

Donors are either MDs or CRDs. For haplo-HCT, young, male siblings or paternal donor is the first choice whereas MD or CRD is the last choice [3, 19, 23]. HLA typing was undertaken according to our previous protocol $[24,25]$.

\section{Transplant procedures}

The conditioning regimen is a standardized ATG/GCSFbased MAC protocol (the so-called Beijing protocol) comprising of the following: cytarabine, $4 \mathrm{~g} / \mathrm{m}^{2} /$ day intravenously (i.v.) on days -10 and -9 ; busulfan (BU), $9.6 \mathrm{mg} / \mathrm{kg}$ i.v., given in 12 doses on days -8 to -6 ; $\mathrm{Cy}, 1.8 \mathrm{~g} / \mathrm{m}^{2} /$ day i.v. on days -5 and -4 ; simustine, $250 \mathrm{mg} / \mathrm{m}^{2}$, orally, on day - 3; and rabbit ATG (Sangstat-Genzyme) $2.5 \mathrm{mg} / \mathrm{kg} /$ day i.v., on days from -5 to -2 . Two doses of $14.5 \mathrm{mg} / \mathrm{kg}$ Cy was given on days 3 and 4 post-HCT in ATG-PTCy cohort according to the trial protocol. Allografts were harvested and infused as previously described [24]. All patients received cyclosporine (CSA), mycophenolate mofetil (MMF), and methotrexate (MTX) for GVHD prophylaxis as detailed in previous report [26]. Methylprednisolone at $1 \mathrm{mg} / \mathrm{kg} /$ day i.v was given to patients developing grade II 
or greater acute GVHD (aGVHD). Steroid refractory aGVHD (SR aGVHD) was defined as progression of GVHD severity after 3 days of steroid therapy or no improvement after 5 days of steroid therapy. Patients with SR aGVHD received second-line treatment with basiliximab (Novartis Pharma AG, Basel, Switzerland) [26]. Details of cytomegalovirus (CMV) and Epstein-Barr virus (EBV) monitoring were described previously [24].

We used two methods for minimal residual disease (MRD) measurement in bone marrow samples at the time of transplant: (1) aberrant leukemia-associated immune phenotypes (LAIPs) detected by four-color flow cytometry (FCM) and (2) WT1 mRNA levels detected by polymerase chain reaction (PCR). FCM-positive was defined as $>0.01 \%$ of cells with a LAIPs phenotype in $\geq$ 1 bone marrow samples. WT1-positive was defined as a transcript level $>0.60 \%$ in $\geq 1$ bone marrow samples. Subjects were defined as MRD positive if they were both FCM and WT1 positive in a single sample [22]. The chimerism of leukocytes was analyzed post-transplant utilizing polymerase chain reaction amplification of short tandem repeats (PCR-STR). Semi-quantitative detection of PCR-STR products was analyzed using denaturing high-performance liquid chromatography (DHPLC) at Peking University before year 2017, and afterward, capillary electrophoresis on ABI PRISM 3130 Genetic Analyzer was used for quantitative detection of PCR-STR while the other two centers consistently adopted the latter one.

\section{Definitions and statistical analysis Study design}

The current study is an extension of our prospective study in which patients received ATG/GCSF-based MAC followed by low-dose PTCy (ATG-PTCy cohort, cohort A) at the Peking University People's Hospital, according to the protocol of clinical trial (registered at http://clinicaltrials.gov/ NCT02412423). Results were compared with a contemporary external control cohort of patients also transplanted from MDs or CRDs who received the identical standardized ATG/GCSF-based MAC protocol (the so-called Beijing protocol, below described in detail), however without the addition of lowdose PTCy (ATG cohort, cohort B) at the first affiliated hospital of Soochow University or Nanfang Hospital, Southern Medical University. The current contemporary external control is distinguished from that in our previous report in which patients transplanted from donors other than MDs or CRDs without low-dose PTCy were analyzed as contemporary control. As all patients transplanted from MDs or CRDs received the combined treatment since the trial started and from then on at Peking University People's Hospital, the other two most experienced transplant centers in China were chosen as external control. The transplant patient number in the three transplant centers ranked top 3 among public hospitals in Chinese Bone Marrow Transplantation Registry (CBMTR). Data for all the patients from the two cohorts are routinely prospectively collected using a predefined case report form.

\section{End points}

The primary study end point was the incidence of acute GVHD, grades III-IV. Secondary end points were the engraftment rate, chimerism, the incidences of acute GVHD grades II-IV, infection, chronic GVHD, the cumulative incidences of NRM and relapse, and probability of disease-free survival (DFS), overall survival (OS), and GVHD-free, relapse-free survival (GRFS).

Definitions of myeloid and platelet recovery, engraftment, graft failure, infection, NRM, relapse, DFS, and OS were detailed previously [25]. Full donor chimerism was defined as $\geq 95 \%$ leukocytes of donor origin in peripheral blood samples [25]. GVHD was defined and graded according to published criteria [27, 28]. Adjudication of GVHD diagnosis used case report forms containing data regarding organ involvement, which were verified by medical record inspection. A panel of five blinded experts determined the presence and grade of GVHD based on these data. GRFS events were defined as grade III-IV aGVHD, chronic GVHD (cGVHD) requiring systemic immunosuppressive treatment, leukemia relapse, or death from any cause during follow-up after allo-HSCT

\section{Statistical analyses}

Groups were compared with the $\chi^{2}$ statistic for categorical variables and the Mann-Whitney test for continuous variables. Competing risk model was used to calculate cumulative incidences, with relapse treated as a competing event for NRM and with death from any cause as a competing risk for engraftment, GVHD, CMV, or EBV reactivation, and relapse. The Kaplan-Meier curves were used to estimate DFS, OS, and GRFS probabilities. Variables in Table 1 were included into a Cox proportional hazard model. Alpha was set at 0.05 . All $P$ values are two-sided. SAS V. 9.3 (SAS Institute Inc., Cary, North Carolina, USA) and SPSS 19.0 (Mathsoft, Seattle, WA, USA) were used for data analyses.

\section{Results \\ Study population}

A total of 239 consecutive patients (ATG-PTCy cohort $=114$, cohort A; ATG cohort $=125$, cohort B) were identified eligible for this study (maternal donors $=178$; collateral relatives $=61$ ). Forty patients in ATG-PTCy cohort transplanted before August 2016 [18] was previously reported and further followed in the current study. 
Table 1 Subject-, disease-, and transplant-related variables

\begin{tabular}{|c|c|c|c|}
\hline Characteristics & ATG-PTCy cohort $(n=114)$ & ATG cohort $(n=125)$ & $P$ value \\
\hline Recipient age (year, median, range) & $27(5-52)$ & $24(4-65)$ & 0.004 \\
\hline HLA-matching (A, B, DR) & & & 0.001 \\
\hline $3 / 6$ & $94(82 \%)$ & $78(62 \%)$ & \\
\hline $4 / 6$ or $5 / 6$ & $20(18 \%)$ & 47 (38\%) & \\
\hline Recipient gender & & & 0.35 \\
\hline Male & $63(55 \%)$ & 77 (62\%) & \\
\hline Female & $51(45 \%)$ & $48(38 \%)$ & \\
\hline Disease type & & & 0.99 \\
\hline Acute myeloid leukemia (AML) & $55(48 \%)$ & $59(47 \%)$ & \\
\hline Acute lymphoblastic leukemia (ALL) & $47(41 \%)$ & $52(42 \%)$ & \\
\hline Myelodysplastic syndromes (MDS) & $9(8 \%)$ & $11(9 \%)$ & \\
\hline Chronic myeloid leukemia (CML) & $3(3 \%)$ & $3(2 \%)$ & \\
\hline Disease risk index & & & 0.20 \\
\hline Low/intermediate & $101(88 \%)$ & $103(83 \%)$ & \\
\hline High/very high & $13(12 \%)$ & $22(17 \%)$ & \\
\hline Minimal residual disease at transplant & & & 0.86 \\
\hline Negative & $93(82 \%)$ & $104(83 \%)$ & \\
\hline Positive & $21(18 \%)$ & $21(17 \%)$ & \\
\hline Donor source & & & 0.19 \\
\hline Mother & $80(70 \%)$ & $98(78 \%)$ & \\
\hline Collateral relatives & $34(30 \%)$ & $27(22 \%)$ & \\
\hline Donor gender male vs female & 27 vs 7 & 20 vs 7 & \\
\hline Donor age (year, median, range) & $46(12-67)$ & $42(10-61)$ & \\
\hline Maternal vs paternal line & 20 vs 14 & 19 vs 8 & \\
\hline Donor gender & & & 0.15 \\
\hline Male & $27(24 \%)$ & $20(16 \%)$ & \\
\hline Female & $87(76 \%)$ & $105(84 \%)$ & \\
\hline Donor-recipient pair & & & 0.20 \\
\hline Female to male & $68(60 \%)$ & $64(51 \%)$ & \\
\hline Others & $46(40 \%)$ & $61(49 \%)$ & \\
\hline Donor age (year, median, range) & $46(12-67)$ & $42(10-61)$ & 0.002 \\
\hline Blood type matching & & & 0.020 \\
\hline Match & $51(45 \%)$ & $75(60 \%)$ & \\
\hline Mismatch & $63(55 \%)$ & $50(40 \%)$ & \\
\hline Median mononuclear cell (range, 108/kg) & $8.2(3.3-11.8)$ & $10.4(3.8-26.1)$ & $<0.001$ \\
\hline Median CD34+ cells (range, $10^{6} / \mathrm{kg}$ ) & $1.9(0.5-14.1))$ & $3.4(1.6-21.2)$ & $<0.001$ \\
\hline Median $\mathrm{CD}^{+}$cells (range, $10^{8} / \mathrm{kg}$ ) & $1.4(0.3-5.7)$ & $1.5(0.3-11.1)$ & 0.63 \\
\hline Year of transplant & & & 0.006 \\
\hline April 2015 to August 2016 & $40(35 \%)$ & $66(53 \%)$ & \\
\hline September 2016 to June 2018 & $74(65 \%)$ & $59(47 \%)$ & \\
\hline Median follow-up (range, days) & $587(57-1399)$ & $682(3-1448)$ & 0.20 \\
\hline Median follow-up in survivors (range, days) & $661(128-1399)$ & $859(230-1448)$ & 0.007 \\
\hline
\end{tabular}


Patient and donor characteristics are shown in Table 1. No significant difference was observed between groups in terms of patients' gender, gender mismatch, disease type, and disease risk index (DRI). However, patients in cohort A had higher percent of 3/6 HLA-A, B, and DR matching and lower percent of $\mathrm{ABO}$ blood type matching with donor as well as lower number of infused mononuclear cells (MNC) as compared to those in cohort B. Patients or donors in cohort $\mathrm{B}$ were 3 and 4 years younger than that in cohort A, respectively. The detailed information on the "collateral relatives" was shown in Table 1.

\section{Low-dose PTCy significantly reduced acute and chronic GvHD}

The 100-day cumulative incidence of grade III-IV aGVHD in cohort A with low-dose PTCy was significantly reduced as compared to that in cohort B without low-dose PTCy ( $5 \%$ vs $18 \% ; P=0.003$ ) and the rate for grade II-IV aGVHD in cohort $\mathrm{A}$ had a trend to be lower than that in cohort B ( $26 \%$ vs $36 \% ; P=0.14)$.

The 2-year cumulative overall incidences of chronic GVHD in cohort A with low-dose PTCy had a trend to be decreased in comparison with that in cohort B without low-dose PTCy ( $30 \%$ vs $44 \% ; P=0.07$ ). However, the rate of moderate-to-severe chronic GVHD in cohort A was comparable to the rate in cohort B $(17 \%$ vs $16 \% ; P=0.71)$.

As shown in Table 2, the use of low-dose PTCy was a significantly protective factor for grade II-IV aGVHD and grade III-IV aGvHD as well as chronic GVHD in multivariate analysis.

\section{Hematopoietic recovery, infection, and transplant outcomes}

All patients achieved myeloid engraftment by day 30 post-HCT except one in the ATG cohort who died of infection at day 11 post-HCT without myeloid recovery. The median value of donor chimerism at day 30 postHCT was 100\% (range, 95-100\%) in cohort A and 97.3\% (range, 73.9-100\%) in cohort B. The median time to myeloid recovery was 3 days shorter in cohort B (12 days, range, 10-17 days) than in cohort A (15 days, range, $7-23$ days; $P<0.001)$. The 100 -day platelet recovery was significantly lower in cohort $A$ than that in cohort B (90\% vs $97 \% ; P=0.003)$. A multivariate analysis indicated that the use of low-dose PTCy significantly delayed myeloid and platelet recovery (Table 2).

The 100-day cumulative incidence of CMV reactivation in ATG-PTCy cohort was significantly higher than that in ATG cohort $(74 \%$ vs $30 \% ; P<0.001)$ while the 100 -day cumulative incidence of CMV disease was comparable between the two cohorts ( $8 \%$ vs $8 \% ; P=0.95)$. The 100 -day cumulative incidence of EBV reactivation and post-transplant lympho-proliferative disorder (PTLD) was
Table 2 Multivariate analyses of outcomes

\begin{tabular}{|c|c|c|}
\hline Outcome & Hazard ratio $(95 \% \mathrm{Cl})$ & $P$ value \\
\hline \multicolumn{3}{|l|}{ ANC recovery } \\
\hline Cohort ATG-PTCy vs ATG & $0.29(0.21-0.41)$ & $<0.001$ \\
\hline \multicolumn{3}{|l|}{ Other significant factors } \\
\hline Mononuclear cell count & $1.09(1.04-1.14)$ & $<0.001$ \\
\hline $\mathrm{CD} 34^{+}$cell count & $1.08(1.01-1.15)$ & 0.012 \\
\hline \multicolumn{3}{|l|}{ Platelet recovery } \\
\hline Cohort ATG-PTCy vs ATG & $0.30(0.21-0.42)$ & $<0.001$ \\
\hline \multicolumn{3}{|l|}{ Other significant factors } \\
\hline Mononuclear cell count & $1.06(1.01-1.11)$ & 0.008 \\
\hline \multicolumn{3}{|l|}{ Acute GvHD $\geq$ grade 2} \\
\hline Cohort ATG-PTCy vs ATG & $0.58(0.35-0.96)$ & 0.036 \\
\hline \multicolumn{3}{|l|}{ Acute GvHD $\geq$ grade 3} \\
\hline Cohort ATG-PTCy vs ATG & $0.28(0.11-0.69)$ & 0.006 \\
\hline \multicolumn{3}{|l|}{ Total chronic GvHD } \\
\hline Cohort ATG-PTCy vs ATG & $0.60(0.38-0.99)$ & 0.047 \\
\hline \multicolumn{3}{|l|}{ Non-relapse mortality } \\
\hline Cohort ATG-PTCy vs ATG & $0.26(0.08-0.75)$ & 0.014 \\
\hline \multicolumn{3}{|l|}{ Disease risk index } \\
\hline Low/int vs high & $0.28(0.12-0.68)$ & 0.005 \\
\hline \multicolumn{3}{|l|}{ Relapse } \\
\hline Cohort ATG-PTCy vs ATG & $0.65(0.28-1.50)$ & 0.31 \\
\hline \multicolumn{3}{|l|}{ Disease-free survival } \\
\hline Cohort ATG-PTCy vs ATG & $0.44(0.22-0.86)$ & 0.016 \\
\hline \multicolumn{3}{|l|}{ Disease risk index } \\
\hline Low/int vs high & $0.51(0.27-0.99)$ & 0.048 \\
\hline \multicolumn{3}{|l|}{ Overall survival } \\
\hline Cohort ATG-PTCy vs ATG & $0.47(0.22-1.01)$ & 0.055 \\
\hline \multicolumn{3}{|l|}{ Disease risk index } \\
\hline Low/int vs high & $0.48(0.23-0.99)$ & 0.048 \\
\hline \multicolumn{3}{|l|}{ GVHD and relapse-free survival } \\
\hline Cohort ATG-PTCy vs ATG & $0.59(0.38-0.92)$ & 0.021 \\
\hline
\end{tabular}

comparable (21\% vs $20 \% ; P=0.83$; and $3 \%$ vs $2 \% ; P=0.57$; respectively).

Up to Jan 31, 2019, the median follow-up time among survivors in cohorts A and B was 661 days (range, 1281399 ) and 859 days (range, 230-1448) post-HCT, respectively $(P=0.007$, Table 1$)$. The 2-year cumulative incidences of relapse were similar among the two cohorts $(13 \%$ vs $14 \%$ for cohort A vs cohort B; $P=0.62)$, and the relapse rate was $15 \%$ and $9 \%(P=0.61)$ in groups $A$ vs $B$ in the subgroup analysis of high-risk disease. The 2-year cumulative incidence of NRM in study cohort A with low-dose PTCy was significantly reduced as compared with that in cohort B $(6 \%$ vs $15 \% ; P=0.045)$. The 2-year probability of DFS had only a trend to be higher 
in cohort A than that in cohort B ( $81 \%$ vs $71 \% ; P=0.06)$ while OS was comparable between the two cohorts $(83 \%$ vs $77 \% ; P=0.18$ ). However, the 2 -year probability of GRFS in study cohort A with low-dose PTCy was significantly improved as compared with that in cohort B (63\% vs $48 \% ; P=0.020$ ). As shown in Table 2 , the use of lowdose PTCy significantly decreased NRM as well as increased DFS and GRFS in cohort A as compared with that in cohort $\mathrm{B}$ in the multivariate analysis.

Grade III-IV aGvHD $(n=3)$ or severe cGVHD $(n=4)$ accounted for $26 \%$ of death in the ATG cohort as compared to none in the ATG-PTCy cohort while disease relapse was the most common cause of death (56\%) in the ATG-PTCy cohort (Table 3). Incidences of GVHD, NRM, and relapse as well as probability of OS and GRFS are shown in Fig. 1.

\section{Discussion}

We compared our novel GVHD prevention strategy (lowdose PTCy in ATG/GCSF-based regimen) with contemporary control patients all transplanted from maternal donors or collateral relatives at particular high GVHD risk $[3,19,20]$. Cumulative incidence of acute GVHD, grade III-IV aGVHD, and chronic GVHD was significantly lower without GVHD-related death in the ATG-PTCy cohort as compared with the control cohort. Reliable engraftment and disease control have also been achieved. As a result, significantly decreased NRM and improved GRFS have been accomplished. Our observations reveal that low-dose PTCy combined with ATG/GCSF-represented conditioning could be a promising new regimen for GVHD prophylaxis and improve outcomes after haplo-HCT.

The current results suggest that the administration of low-dose PTCy along with ATG/GCSF significantly decreased the rate of both acute and chronic GVHD as compared to contemporary controls who were also transplanted from MDs or CRDs proved to be at particular high GVHD risk under "Beijing protocol" in both single-center and multi-center studies [3, 5, 6, 19, 20]. Furthermore, grade III-IV aGvHD or severe cGVHD GVHD was the main cause of death in 7 out of 27 patients in the control cohort, in contrast to none in the

Table 3 Causes of death

\begin{tabular}{lll}
\hline Cause of death & $\begin{array}{l}\text { ATG-PTCy cohort } \\
(n=16)\end{array}$ & $\begin{array}{l}\text { ATG cohort } \\
(n=27)\end{array}$ \\
\hline Relapse & $9(56 \%)$ & $8(30 \%)$ \\
GVHD & 0 & $7(26 \%)$ \\
$\begin{array}{l}\text { Infection other than } \\
\text { CMV/EBV }\end{array}$ & $5(31 \%)$ & $10(37 \%)$ \\
CMV disease & $1(6 \%)$ & 0 \\
Organ failure & $1(6 \%)$ & $2(7 \%)$ \\
\hline
\end{tabular}

combined treatment cohort (Table 3). It should be stated that despite the significantly decreased rate of grade IIIIV aGvHD and total chronic GVHD in the ATG-PTCy cohort compared to control cohort, the incidence for moderate to severe chronic GVHD remains higher. And the rate of moderate to severe chronic GVHD was also higher or comparable than that in the high-dose PTCy plus ATG protocols reported to be $0-18.8 \%$ with smaller study population or shorter follow-up [12-14, 17]. MAC and predominantly female donors especially female-tomale pairs [3] in the current study may be the attributable reasons as compared with previous reports on high-dose PTCy plus low-dose ATG regimens [12-14]. More recently, Wachsmuth et al. [29] showed that the dose of PTCy is important, with tested doses between 10 and 50 $\mathrm{mg} / \mathrm{kg} /$ day at days +3 and +4 effectively prevented fatal GVHD and $25 \mathrm{mg} / \mathrm{kg} /$ day being the optimal dose in their model while doses outside this range $(\leq 5$ or $\geq 100 \mathrm{mg} / \mathrm{kg} /$ day) proved ineffective in preventing mortality. Overall, our current results verify the feasibility of low-dose PTCy adding to the backbone of ATG/GCSF-based regimen, and this combination for GVHD prevention may represent a promising strategy.

Understanding the mechanism of low-dose PTCy in combination with ATG is vital to explore its maneuver in innovative procedure. In our previous reported prospective trial, data suggested that low-dose PTCy is sufficient to reduce acute GVHD in mouse model, partly due to the enhancement of quick Treg reconstitution. Besides, lowdose PTCy could promote the protective effect of ATG/ G-CSF on GVHD both in mouse and human sets [18]. Additionally, the timing and sequential order of given drugs may be crucial to the synergistic effect. It is conceivable that in spite of slow donor T cell depletion by ATG, donor $\mathrm{T}$ cells escape blockade leading to aGVHD occurrence. These escaped donor $\mathrm{T}$ cells exponentially expand to give rise to aGVHD when exposed to recipient antigens. Wachsmuth et al. [29] proved that PTCy induced alloreactive $\mathrm{T}$ cell dysfunction and suppression and supported the increasingly important role of Tregs over time post-HCT in the amelioration of chronic GVHD. Nonetheless, centers using PBSC in haploidentical transplants with PTCy have shown rates of extensive chronic GVHD as high as $38 \%$ [30]. Even with the addition of $4.5-5 \mathrm{mg} /$ $\mathrm{kg}$ ATG to a backbone of high-dose PTCy, the rate of moderate to severe chronic GVHD was reported to be $10-13 \%$ with 2 -day PTCy $[13,14]$ or $18.8 \%$ with 1 -day PTCy [17]. This may partly explain why only total cGVHD was reduced with the current combined treatment while moderate to severe cGVHD was not. Future randomized studies including data on immune reconstitution and Treg levels will aid to further elucidate the biological aspects.

In the previous report on the combination of ATG and high-dose PTCy, the primary rejection rate was $8 \%$ 


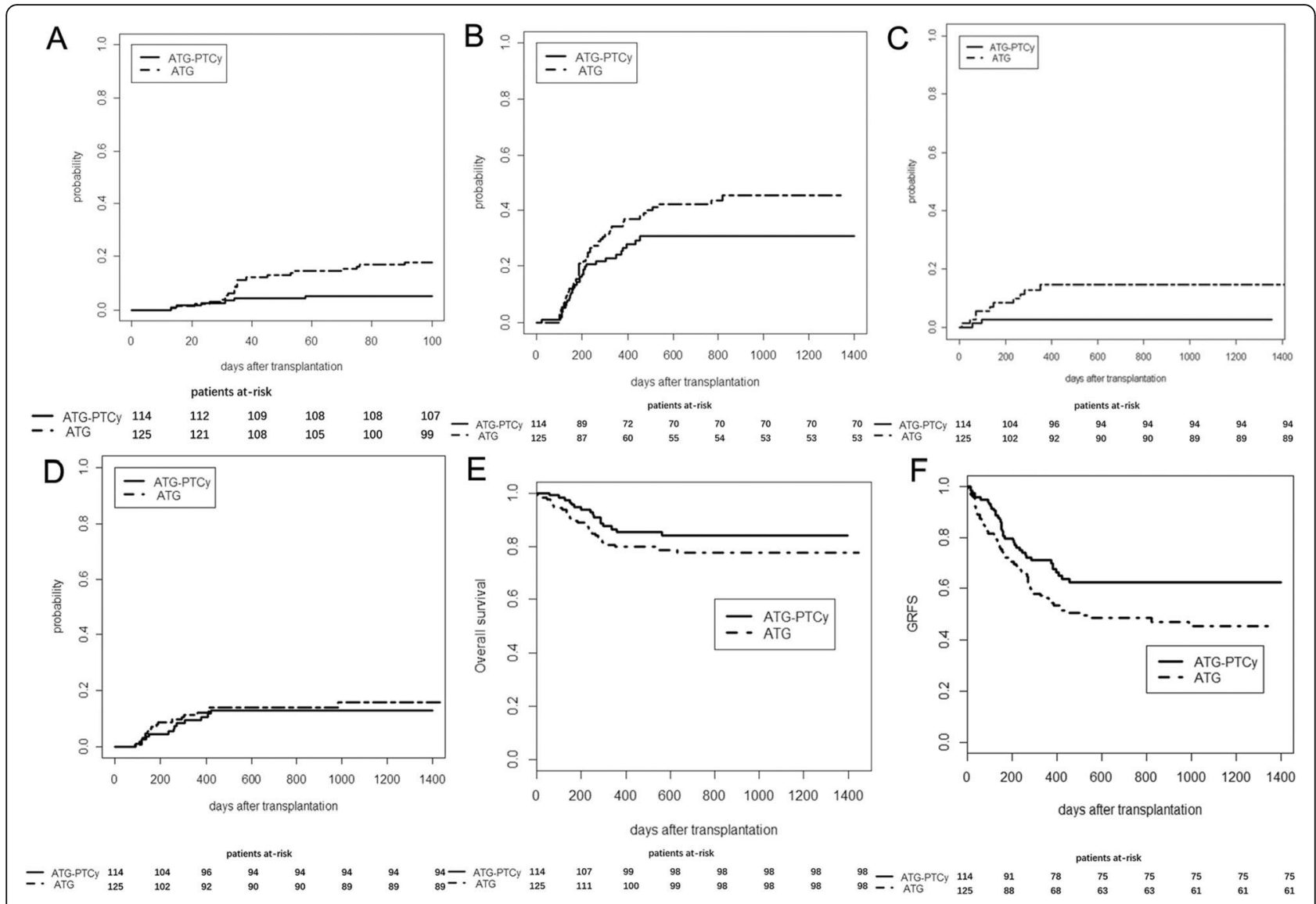

Fig. 1 Rates of graft-versus-host disease (GVHD), non-relapse-mortality (NRM), relapse incidence (RI), overall survival (OS), and GVHD-free, relapsefree survival (GRFS) according to treatment group. Rates of grades III-IV acute GVHD (a), chronic GVHD (b), NRM (c), RI (d), OS (e), and GRFS (f)

[11]. In the current study, although the myeloid engraftment was $100 \%$, hematopoietic recovery using low-dose PTCy along with ATG was slower than that in the control group. It may partly due to the immediate allo-response in which alloreactive $\mathrm{T}$ cells were indicated to dominate early after PTCy. It was documented that the inclusion of ATG in conditioning regimens yields quicker achievement of donor chimerism [31], and ATG was suggested to be capable to aid reliable engraftment by decreasing the aforementioned immediate allo-response early after PTCy [29]. As for disease control, it is speculated that low-dose PTCy had little impact on relapse, which is evidenced by our current data. Previous studies also demonstrated that high-dose PTCy with ATG is effective in alleviating GVHD without increasing relapse [13, 14] with the relapse incidences reported to be $16-37 \%[11-14,17]$.

It was taken in the context of the fact that the combination of ATG and PTCy appears to increase infectious complications particularly regarding herpes virus control and may lose some of the protective effects of PTCy alone. Higher rate of CMV reactivation was noted in the combined treatment cohort as compared to the control cohort although the rate was similar to the previous report on the joint use of high-dose PTCy and ATG (74\%). As compared with the control cohort, $\mathrm{T}$ cell impairment between donor and recipient secondary to higher extent of HLA mismatch and the dual $\mathrm{T}$ cell depletion with PTCy and ATG may contribute to the higher incidence of CMV reactivation. Nonetheless, only one CMV-related death occurred (Table 3). We have implemented a preemptive CMV-CTL strategy with the aim to alleviate the CMV infection risk. As for the observation that low-dose PTCy increased the risk of CMV reactivation but not the risk of relapse, although it looks in contrast to the hypothesis that PTCy acts partly by eliminating activated alloreactive $\mathrm{T}$ cells, it may be partly explained by recent report that CMV reactivation and expansion of $\mathrm{CD} 56^{\text {bright }} \mathrm{CD} 16^{\mathrm{dim} /-}$ DNAM1+ natural killer cells are associated with antileukemia effect after haploidentical HCT in acute leukemia [32, 33]. In addition, Wachsmuth et al. [29] revealed that $25 \mathrm{mg} / \mathrm{kg}$ PTCy did not significantly reduce CD8+ $\mathrm{T}$ cell proliferation while the $\mathrm{T}$ cell reconstitution after CMV reactivation under the combined treatment strategy needs to be elucidated. One of the characteristics of the PTCy studies has been a reduced incidence or even absence of 
PTLD [34]. Though we found EBV reactivation incidence was $21 \%$, it was remarkably less in comparison with other studies using high-dose PTCy and ATG (32-64\%) [11-14, 17]. In order to reduce the infectious complications, the next step with testing ATG/PTCy (low-dose) strategy may be decreasing the ATG dose also.

The limitation of the study is the non-randomized feature. We did matched-pair analysis to balance characteristics of the two populations, and the matched-pair analysis confirmed the main outcome results that we found in the standard analysis (data not shown due to the small population). Another limitation is that the comparison here is across institutions. Although the identical ATG/ GCSF-based "Beijing protocol" is adopted in all the three centers (the largest and the most experienced transplant centers in China as described in the "Patients and methods" section), one cannot completely rule out that there exist small differences of medical practices between participating centers which may partly contribute to the transplant outcomes. Nevertheless, since we extended the prospective trial with the combined treatment for all HCT from MDs or CRDs in Peking University, the contemporaneous external control also transplanted from MDs or CRDs can only come from other centers. Multi-center randomized trials in different transplant settings and dose-finding studies including decreasing the ATG dose are needed to extend the practical use of the new combined treatment strategy.

In conclusion, our results showed that $\mathrm{T}$ cell-replete haplo-HCT after ATG/GCSF-based intensified conditioning combined with low-dose PTCy is a feasible and effective protocol, especially for patients at particular high GVHD risk. This strategy produced reliable donor cell engraftment with low rates of GVHD while keeping disease control.

\section{Abbreviations}

aGVHD: Acute graft-versus-host disease; AL: Acute leukemia; ATG: Antithymocyte globulin; BU: Busulfan; CGVHD: Chronic graft-versus-host disease; CML: Chronic myeloid leukemia; CMV: Cytomegalovirus; CRD: Collateral relative donor; CSA: Cyclosporine; DFS: Disease-free survival; EBV: Epstein-Barr virus; GCSF: Granulocyte colony-stimulating factor; GRFS: GVHD-free, relapsefree survival; GVHD: Graft-versus-host disease; haplo-HCT: Haploidentical donor hematopoietic cell transplant; MAC: Myeloablative conditioning; MD: Mother donor; MDS: Myelodysplastic syndrome; MMF: Mycophenolate mofetil; MTX: Methotrexate; NRM: Non-relapse mortality; OS: Overall survival; PTCy: Post-transplant cyclophosphamide; TCR: T cell-replete; Treg: Regulatory T cell; URD: Unrelated donor

\section{Acknowledgements}

We thank the principal investigators and the skilled teams.

\section{Authors' contributions}

$\mathrm{XJH}$ designed the research; $\mathrm{YW}$ and $\mathrm{XJH}$ analyzed the data and wrote the manuscript; and all authors provided patient data and gave final approval for the manuscript. The authors declare no competing financial interests.

\section{Funding}

This work was partly supported by grants from the National Key Research and Development Program of China (2017YFA0104500) from the Ministry of
Science and Technology, National Natural Science Foundation of China (Grant No. 81770189 \& 81621001 \&81530046 \&81670167), Collaborative Innovation Center of Hematology China, the Science and Technology Project of Guangdong Province of China (Grant No. 2016B030230003), Peking University Clinical Scientist Program(BMU2019LCKXJ003), the Fundamental Research Funds for the Central Universities, and the project of health collaborative innovation of Guangzhou city (no. 201704020214).

\section{Availability of data and materials}

The datasets used and/or analyzed during the current study are available from the corresponding author on reasonable request.

\section{Ethics approval and consent to participate}

The study protocol was approved by the Ethics Committee of Peking University People's Hospital, the first affiliated hospital of Soochow University, and Nanfang Hospital. The study is registered at http://clinicaltrials.gov/ NCT02412423. Informed consent was obtained from each patient.

\section{Consent for publication}

Not applicable.

\section{Competing interests}

The authors declare that they have no competing interests.

\section{Author details}

'Peking University People's Hospital, Peking University Institute of Hematology, National Clinical Research Center for Hematologic Disease, Beijing Key Laboratory of Hematopoietic Stem Cell Transplantation, No.11, Xizhimen South Street, Xicheng District, Beijing 100044, China. ${ }^{2}$ The first affiliated hospital of Soochow University, Suzhou 215006, Jiangsu, China. ${ }^{3}$ Nanfang Hospital, Southern Medical University, Guangzhou 510515, Guangdong, China. ${ }^{4}$ Peking-Tsinghua Center for Life Sciences, Beijing 100871, China.

Received: 10 July 2019 Accepted: 23 August 2019

Published online: 03 September 2019

\section{References}

1. Apperley J, Niederwieser D, Huang XJ, Nagler A, Fuchs E, Szer J, et al. Haploidentical hematopoietic stem cell transplantation: a global overview comparing Asia, the European Union, and the United States. Biol Blood Marrow Transplant. 2016;22:23-6.

2. Rubio MT, Savani BN, Labopin M, Piemontese S, Polge E, Ciceri F, et al. Impact of conditioning intensity in T-replete haplo-identical stem cell transplantation for acute leukemia: a report from the acute leukemia working party of the EBMT. J Hematol Oncol. 2016;9:25.

3. Wang Y, Chang YJ, Xu LP, Liu KY, Liu DH, Zhang XH, et al. Who is the best donor for a related HLA haplotype-mismatched transplant? Blood. 2014;124: $843-50$

4. Wang Y, Liu QF, Xu LP, Liu KY, Zhang XH, Ma X, et al. Haploidentical vs identical-sibling transplant for AML in remission: a multicenter, prospective study. Blood. 2015;125:3956-62.

5. Wang $Y$, Liu $Q F, X u L P$, Liu KY, Zhang $X H, M a X$, et al. Haploidentical versus matched-sibling transplant in adults with Philadelphia-negative high-risk acute lymphoblastic leukemia: a biologically phase III randomized study. Clin Cancer Res. 2016;22:3467-76.

6. Wang Y, Wang HX, Lai YR, Sun ZM, Wu DP, Jiang M, et al. Haploidentical transplant for myelodysplastic syndrome: registry-based comparison with identical sibling transplant. Leukemia. 2016;30:2055-63.

7. Robinson TM, O'Donnell PV, Fuchs EJ, Luznik L. Haploidentical bone marrow and stem cell transplantation: experience with post-transplantation cyclophosphamide. Semin Hematol. 2016:53:90-7.

8. Ciurea SO, Zhang MJ, Bacigalupo AA, Bashey A, Appelbaum FR, Aljitawi OS, et al. Haploidentical transplant with posttransplant cyclophosphamide vs matched unrelated donor transplant for acute myeloid leukemia. Blood. 2015;126:1033-40.

9. Kasamon YL, Bolanos-Meade J, Prince GT, Tsai HL, McCurdy SR, Kanakry JA, et al. Outcomes of nonmyeloablative HLA-haploidentical blood or marrow transplantation with high-dose post-transplantation cyclophosphamide in older adults. J Clin Oncol. 2015;33:3152-61. 
10. Bolanos-Meade J, Fuchs EJ, Luznik L, Lanzkron SM, Gamper CJ, Jones RJ, et al. HLA-haploidentical bone marrow transplantation with posttransplant cyclophosphamide expands the donor pool for patients with sickle cell disease. Blood. 2012;120:4285-91.

11. Law AD, Salas MQ, Lam W, Michelis FV, Thyagu S, Kim DDH, et al. Reducedintensity conditioning and dual T lymphocyte suppression with antithymocyte globulin and post-transplant cyclophosphamide as graft-versus-host disease prophylaxis in haploidentical hematopoietic stem cell transplants for hematological malignancies. Biol Blood Marrow Transplant. 2018;24:2259-64.

12. Dulery R, Menard AL, Chantepie S, El-Cheikh J, Francois S, Delage J, et al. Sequential conditioning with thiotepa in T cell- replete hematopoietic stem cell transplantation for the treatment of refractory hematologic malignancies: comparison with matched related, haplo-mismatched, and unrelated donors. Biol Blood Marrow Transplant. 2018:24:1013-21.

13. Dawsari G, Hassanein M, Rasheed W, Almohareb F, Chaudhri NA, Alsharif F, et al. Addition of ATG to myeloablative haplo conditioning with post-transplantation cyclophosphamide might decrease the risk of GVHD and TRM without increasing the risk of relapse. Blood. 2016;128:5871.

14. Lin CC, Chen TT, Lo WJ, Lein MY, Hiseh CY, Lin CY, et al. Post-transplant cyclophosphamide (PTCy) with anti-thymocyte globulin (ATG) as GVHD prophylaxis is effective in haploidentical peripheral stem cell transplantation and without increasing risk of relapse. Blood. 2017;130:1978.

15. Prem S, Atenafu EG, Al-Shaibani Z, Loach D, Law A, Lam W, et al. Low rates of acute and chronic GVHD with ATG and PTCy in matched and mismatched unrelated donor peripheral blood stem cell transplants. Eur J Haematol. 2019;102:486-93.

16. Deotare U, Atenafu EG, Loach D, Michelis FV, Kim DD, Thyagu S, et al. Reduction of severe acute graft-versus-host disease using a combination of pre transplant anti-thymocyte globulin and post-transplant cyclophosphamide in matched unrelated donor transplantation. Bone Marrow Transplant. 2018;53:361-5.

17. Yang J, Jiang J, Cai Y, Li S, Wan L, Zhu J, et al. Low-dose anti-thymocyte globulin plus low-dose posttransplant cyclophosphamide as graft-versushost disease prophylaxis in haploidentical peripheral blood stem cell transplantation combined with unrelated cord blood for patients with hematologic malignancies: a prospective, phase II study. Bone Marrow Transplant. 2019:54:1049-57.

18. Wang Y, Chang YJ, Chen L, Xu LP, Bian ZL, Zhang XH, et al. Low-dose posttransplant cyclophosphamide can mitigate GVHD and enhance the G-CSF/ ATG induced GVHD protective activity and improve haploidentical transplant outcomes. Oncoimmunology. 2017;6:e1356152.

19. Zhang YY, Liu DH, Liu KY, Xu LP, Chen H, Han W, et al. HLA-haploidentical hematopoietic SCT from collateral related donors without in vitro T-cell depletion for hematological malignancies. Bone Marrow Transplant. 2014;49:496-501.

20. Mo XD, Zhang YY, Zhang XH, Xu LP, Wang Y, Yan CH, et al. The role of collateral related donors in haploidentical hematopoietic stem cell transplantation. Science Bulletin. 2018;63:1376-82.

21. Armand P, Gibson CJ, Cutler C, Ho VT, Koreth J, Alyea EP, et al. A disease risk index for patients undergoing allogeneic stem cell transplantation. Blood. 2012;120:905-13.

22. Wang $\mathrm{Y}$, Chen $\mathrm{H}$, Chen J, Han $\mathrm{M}, \mathrm{Hu}$ J, Jiong $\mathrm{H}$, et al. The consensus on the monitoring, treatment, and prevention of leukemia relapse after allogeneic hematopoietic stem cell transplantation in China. Cancer Lett. 2018;438:63-75.

23. Xu L, Chen $\mathrm{H}$, Chen J, Han M, Huang H, Lai $Y$, et al. The consensus on indications, conditioning regimen, and donor selection of allogeneic hematopoietic cell transplantation for hematological diseases in China-recommendations from the Chinese Society of Hematology. Hematol Oncol. 2018;11:33

24. Wang Y, Fu HX, Liu DH, Xu LP, Zhang XH, Chang YJ, et al. Influence of two different doses of antithymocyte globulin in patients with standard-risk disease following haploidentical transplantation: a randomized trial. Bone Marrow Transplant. 2014;49:426-33.

25. Chang $Y$ J, Zhao XY, Xu LP, Zhang XH, Wang $Y$, Han W, et al. Donor-specific anti-human leukocyte antigen antibodies were associated with primary graft failure after unmanipulated haploidentical blood and marrow transplantation: a prospective study with randomly assigned training and validation sets. J Hematol Oncol. 2015;8:84.

26. Yan $\mathrm{CH}$, Wang $\mathrm{Y}$, Wang JZ, Chen YH, Chen Y, Wang FR, et al. Minimal residual disease- and graft-vs-host disease-guided multiple consolidation chemotherapy and donor lymphocyte infusion prevent second acute leukemia relapse after allotransplant. J Hematol Oncol. 2016;9:87.

27. Rowlings PA, Przepiorka D, Klein JP, Gale RP, Passweg JR, Henslee-Downey PJ, et al. IBMTR Severity Index for grading acute graft-versus-host disease: retrospective comparison with Glucksberg grade. Br J Haematol. 1997;97:855-64.

28. Lee SJ, Wolff D, Kitko C, Koreth J, Inamoto Y, Jagasia M, et al. Measuring therapeutic response in chronic graft-versus-host disease. National Institutes of Health consensus development project on criteria for clinical trials in chronic graft-versus-host disease: IV. The 2014 Response Criteria Working Group report. Biol Blood Marrow Transplant. 2015;21:984-99.

29. Wachsmuth LP, Patterson MT, Eckhaus MA, Venzon DJ, Gress RE, Kanakry CG. Post-transplantation cyclophosphamide prevents graft-versus-host disease by inducing alloreactive T cell dysfunction and suppression. J Clin Invest. 2019;130:2357-73.

30. Bashey A, Zhang X, Sizemore CA, Manion K, Brown S, Holland HK, et al. T-cell-replete HLA-haploidentical hematopoietic transplantation for hematologic malignancies using posttransplantation cyclophosphamide results in outcomes equivalent to those of contemporaneous HLA-matched related and unrelated donor transplantation. J Clin Oncol. 2013;31:1310-6.

31. Colson YL, Li H, Boggs SS, Patrene KD, Johnson PC, Ildstad ST. Durable mixed allogeneic chimerism and tolerance by a nonlethal radiation-based cytoreductive approach. J Immunol. 1996:157:2820-9.

32. Jang JE, Hwang DY, Chung H, Kim SJ, Eom Jl, Jeung HK, et al. Early cytomegalovirus reactivation and expansion of CD56brightCD16dim/ -DNAM1+ natural killer cells are associated with antileukemia effect after haploidentical stem cell transplantation in acute leukemia. Biol Blood Marrow Transplant. 2019. https://doi.org/10.1016/j.bbmt.2019.06.008.

33. Hu LJ, Cao XH, Yu XX, Liu XF, Zhao XS, Chang YJ, et al. NK cell reconstitution following unmanipulated HLA-mismatched/haploidentical transplantation compared with matched sibling transplantation. Sci China Life Sci. 2019. https://doi.org/10.1007/s11427-018-9565-5.

34. Kanakry JA, Kasamon YL, Bolanos-Meade J, Borrello IM, Brodsky RA, Fuchs EJ, et al. Absence of post-transplantation lymphoproliferative disorder after allogeneic blood or marrow transplantation using post-transplantation cyclophosphamide as graft-versus-host disease prophylaxis. Biol Blood Marrow Transplant. 2013;19:1514-7.

\section{Publisher's Note}

Springer Nature remains neutral with regard to jurisdictional claims in published maps and institutional affiliations.

\section{Ready to submit your research? Choose BMC and benefit from:}

- fast, convenient online submission

- thorough peer review by experienced researchers in your field

- rapid publication on acceptance

- support for research data, including large and complex data types

- gold Open Access which fosters wider collaboration and increased citations

- maximum visibility for your research: over $100 \mathrm{M}$ website views per year

At BMC, research is always in progress.

Learn more biomedcentral.com/submissions 